\title{
Differences in output from corticosteroid inhalers used with a volumatic spacer
}

\author{
M.R. Miller, P. Bright
}

Accepted after revision May 181995

Differences in output from corticosteroid inhalers used with a volumatic spacer. M.R. Miller, P. Bright. CERS Journals Ltd 1995.

ABSTRACT: Corticosteroid inhaler therapy using a spacer device is commonly used as an important part of asthma management. Increasingly, generic corticosteroid inhalers are being used with spacer devices. We have therefore tested whether these generic inhalers yield equivalence in dose when compared to the established inhalers.

We measured the in vitro output, discharged into a Volumatic spacer from beclomethasone dipropionate inhalers $\left(250 \mu \mathrm{g}^{\left.\cdot \text { puff }^{-1}\right)}\right.$ made by three manufacturers, Allen \& Hanburys, 3M and Baker Norton. The output from 20 of each type of inhaler was sampled, in random order, by a computer driven pump system. Beclomethasone was absorbed onto a coded filter, which was analysed independently for drug content.

The output per puff differed significantly between the inhalers of each manufacturer, with a $36 \%$ difference between the highest output from the Allen \& Hanburys device and the lowest output device.

We conclude that there are important differences in output from these inhalers when used with a spacer, and that substitution of one device with another will not

Dept of Medicine, University of Birmingham, Good Hope NHS Trust, Sutton Coldfield, UK.

Correspondence: M.R. Miller

Dept of Medicine

University of Birmingham

Good Hope NHS Trust

Sutton Coldfield

West Midlands B75 7RR

UK

Keywords: asthma

beclomethasone dipropionate

corticosteroids

inhaler therapy

Received: March 71995 necessarily give equivalent therapy to the patient.

Eur Respir J., 1995, 8, 1637-1638.

With the introduction of generic inhalers, it has become practice to use them with the currently available spacer devices, such as the Volumatic spacer (Allen \& Hanburys Ltd, UK). We have studied whether the output of three different inhalers, delivering $250 \mu \mathrm{g}$ of beclomethasone dipropionate (BDP) per puff, was the same when they are used with a Volumatic spacer.

\section{Methods}

Twenty metered-dose inhalers of BDP $(250 \mu \mathrm{g}$ per actuation) from each of three different manufacturers, Allen \& Hanburys (A\&H), Baker Norton (BN) and 3M were purchased from a commercial pharmaceutical wholesaler. The output from each inhaler when used with a Volumatic spacer was tested using the following protocol. The 60 inhalers were tested in a mixed and random order. Each inhaler was shaken by hand and then held still and upright for $2 \mathrm{~s}$ before discharging one puff into the atmosphere. This was repeated a total of 5 times. The inhaler was then inserted into a Volumatic spacer that had been washed in a dilute soapy solution, rinsed in benzalkonium chloride, wiped with chlorhexidine and $70 \%$ spirit cloth, and dried without rubbing. Prior to use, each spacer was checked using a static electricity meter (Farnell Electronic Components, Leeds, UK; Part
No. 212-880) to be free from static electricity, which might attract airborne particles.

The mouthpiece of the Volumatic was attached to a Respirgard II filter assembly (Brunner Scientific, Hunmanby, North Yorks, UK), which can remove all the airborne drug particles in one pass. The filter assembly comprised a clear plastic casing, which held the filter in place, and each was supplied with the assembly stored prior to use in a polythene bag to prevent any contamination or collection of static electricity. Each filter assembly was removed from its bag immediately prior to use to be attached at one end to the Volumatic spacer and at the other end to a computer-controlled pump, which was programmed to withdraw $1,000 \mathrm{~mL}$ from the spacer at a constant flow of $1 \mathrm{~L} \cdot \mathrm{s}^{-1}$. Figure 1 shows the configuration of the inhaler, spacer, and filter assembly.

Each inhaler was discharged once into the spacer, and after a $2 \mathrm{~s}$ delay the pump drew 1,000 $\mathrm{mL}$ of air through the system. This was immediately repeated a second

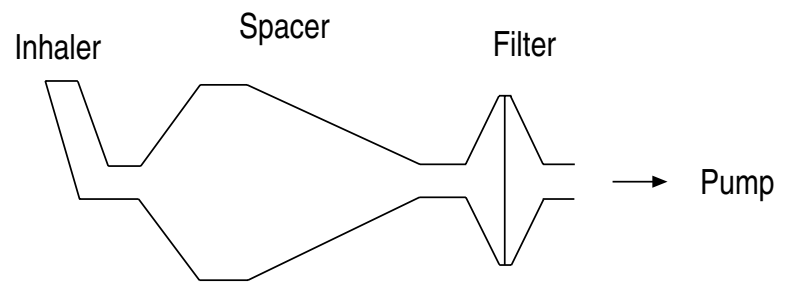

Fig. 1. - The configuration of the inhaler, spacer, and filter assembly when ready for testing. 
time and the filter then removed, coded, and sealed back in its polyethylene bag. After the final of the five pretest puffs, there was a lapse of $10 \mathrm{~s}$ for the attachment of the spacer before the discharge of the first test puff. Two seconds elapsed before the pump "inhaled" the dose over $1 \mathrm{~s}$. A further $15 \mathrm{~s}$ elapsed whilst the filter and spacer assembly was disconnected from the pump, to allow the pump to be returned to its start position and the assembly reconnected again. The second puff was then delivered into the spacer, and a further $2 \mathrm{~s}$ delay occurred before the pump inhaled the second dose over $1 \mathrm{~s}$. After each run with an inhaler, the spacer was removed and washed as above. The 60 coded filters were sent to an independent commercial chemical analyst for assay of BDP using reversed phase high performance liquid chromatography. The filter codes were broken after the results of the analysis were returned.

Statistical procedures were undertaken using the Statistical Package for the Social Sciences for Windows Release $6.0,1993$. A probability of less than $5 \%$ was taken as significant.

\section{Results}

The individual results which represent the retrieved BDP from two puffs of each inhaler when used with a Volumatic spacer are shown in figure 2. The variances of the mean outputs from the 20 inhalers of each of the three manufacturers were not significantly different from one another (Levene test, $\mathrm{p}=0.75$ ), and the means, ranges, minimum and maximum values are shown in table 1 . The means were significantly different from each other (one-way analysis of variance, $\mathrm{p}<0.0001$ ), with the mean dose delivered by the inhaler with the lowest yield being only $64 \%$ of that of the inhaler with the highest yield. The differences between each of the pairs of means were significantly different from zero (Bonferroni's test for multiple comparisons, $\mathrm{p}<0.05)$, indicating that the output of the inhalers

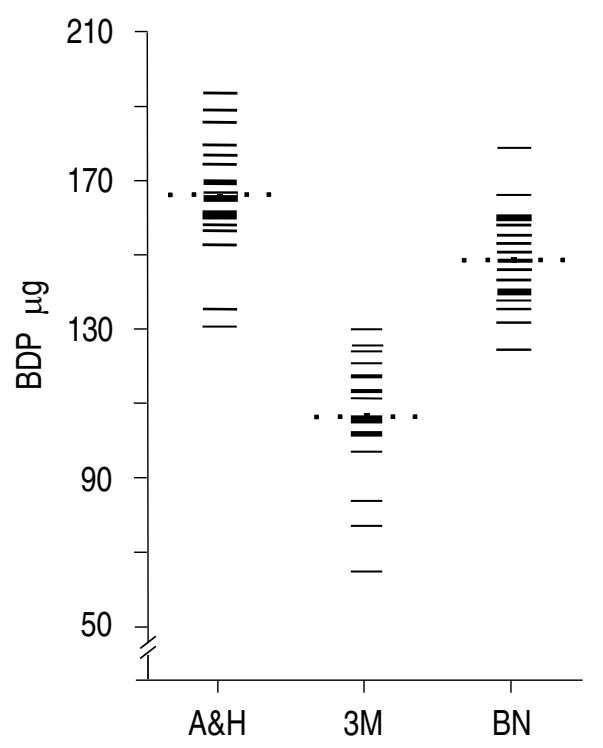

Fig. 2. - The output for two puffs from each of the 20 inhalers tested for the three manufacturers, Allen \& Hanburys (A\&H), Baker Norton $(\mathrm{BN})$ and $3 \mathrm{M}$. Mean values for each manufacturer are shown by a horizontal dotted line. BDP: beclomethasone dipropionate.
Table 1. - Data for 20 of each type of inhaler expressed as the output in micrograms for two puffs

\begin{tabular}{|c|c|c|c|}
\hline & $\mathrm{A} \& \mathrm{H}$ & $\mathrm{BN}$ & $3 \mathrm{M}$ \\
\hline Mean outputs $\mu \mathrm{g}$ & $166.1(3.5)$ & $149.0(2.9)$ & $106.8(3.7)$ \\
\hline $\operatorname{Min} \mu \mathrm{g}$ & 130.8 & 125.0 & 65.1 \\
\hline $\operatorname{Max} \mu \mathrm{g}$ & 193.6 & 179.0 & 130.2 \\
\hline Max-Min & 62.8 & 54.0 & 65.1 \\
\hline
\end{tabular}

Numbers in brackets are SEM. A\&H: Allen \& Hanburys; BN: Baker Norton. Max: maximum; Min: minimum.

from each manufacturer was significantly different from that of the other two manufacturers.

\section{Discussion}

The British Thoracic Society guidelines for the management of asthma recommend that high dose inhaled corticosteroids should be used with spacer devices [1]. Recent correspondence has expressed concern that delivery of BDP from Volumatic spacers may differ depending on the type of inhaler used [2-4]. We have conducted a rigorously controlled in vitro study to reduce all confounding factors, such as static electricity and shape of inhalation profile, so that valid comparisons can be made between the different inhalers. The differences found in inhaler output indicate that the cost advantage of using generic inhalers with spacer devices may be misleading, as patients may be receiving less treatment than envisaged by the clinician.

These findings could be due to the particle size distribution delivered by the three inhalers. It has been shown that the total respirable fraction is about the same between the three manufacturers [4], but the A\&H product has the highest proportion of smaller particles, with the $3 \mathrm{M}$ product having the highest proportion of larger particles, which will undergo greater sedimentation due to gravity within the spacer device. The possible confounding effect of static electricity is important, since smaller particles may be more susceptible to this effect, so that experiments that have not taken this into account could potentially bias the result.

We conclude that equivalent dosing of BDP from generic inhalers when used with a Volumatic spacer cannot be assumed, and so simple substitution of one inhaler with another will not necessarily give equivalent therapy to the patient. It is essential that when new inhaler devices are made available for patient use they must have been adequately tested in all the possible ways they may be used by patients. Only then will fully informed prescribing be ensured.

Acknowledgements: The authors acknowledge that the costs incurred in purchasing the inhalers and obtaining the independent chemical analysis have been met by Allen \& Hanburys.

\section{References}

1. Guidelines on the management of asthma. Statement by the British Thoracic Society. Thorax 1993; 48 (Suppl.): S1-S24.

2. Pearson M, Lewis R, Watson $\mathrm{J}$, et al. Generic inhalers for asthma. $B r$ Med J 1994; 309: 1440.

3. Snell NJC. Substantial differences. Pharmaceut J 1994; 253: 330 . 